that this is because closed-cell stents have a smaller free cell area, restricting plaque protrusion (PP). Reducing the free cell area of stents may thus reduce the incidence of ischemic complications. We examine whether the use of overlapped (i.e., double) closedcell stents in CAS for carotid artery stenosis with unstable plaque might inhibit plaque protrusion (PP).

Methods 41 consecutive patients with carotid artery stenosis with unstable plaque diagnosed by magnetic resonance plaque imaging (35 men; [mean 76.3 years]; symptomatic stenosis, 28 lesions; mean stenosis severity, 84.8\%) were prospectively analyzed. All CAS procedures were performed by conservative post-dilatation after stent-in-stent placement of two Carotid Wallstents using an embolic protection device. The technical success rate and the incidence of PP, ischemic stroke within 30 days, and new ipsilateral ischemic lesions on diffusionweighted imaging (DWI) within $48 \mathrm{~h}$ after CAS and follow-up results (ipsilateral stroke rate and restenosis rate) were prospectively assessed.

Results The technical success rate was 100\% (41/41). No PP and stroke occurred in any patients. New ischemic lesions were observed on DWI in 11 patients (26.8\%). During the follow-up period (mean 11.6 months), no ipsilateral strokes occurred. Asymptomatic restenosis (53\%) occurred in 1 patient (2.9\%); asymptomatic occlusion occurred in 1 patient (2.9\%).

Conclusion CAS using overlapped double stents for unstable plaque may be useful for preventing PP and ischemic complications.

Disclosures K. Takayama: None. K. Myouchin: None. T. Wada: None. S. Kurokawa: None. K. Kichikawa: None.

\section{P-002 SINGLE SHOT INTRA-AORTIC ANGIOGRAPHY FOR WHOLE BRAIN VASCULAR AND PERFUSION IMAGING UTILIZING A HYBRID CT-ANGIOGRAPHY SUITE - POTENTIAL APPLICATION IN ENDOVASCULAR STROKE THERAPY}

${ }^{1} \mathrm{~A}$ Rai ${ }^{*},{ }^{2} \mathrm{~A}$ Tarabishy, ${ }^{1} \mathrm{~S}$ Boo. 'Interventional Neuroradiology, West Virginia University, Morgantown, WV; ${ }^{2}$ Neuroradiology, West Virginia University, Morgantown, WV

\subsection{6/neurintsurg-2019-SNIS.38}

Objective To report the feasibility of a combined imaging and treatment strategy for acute ischemic stroke (AIS) utilizing a hybrid CT-Angiography suite.

Methods A descriptive case report covering the technical aspects of whole-brain vascular and perfusion imaging with a single intra-aortic injection of $7 \mathrm{ml}$ of iodinated contrast for suspected large vessel occlusion (LVO).

Results A middle-aged man was transferred after 20-hours with a suspected LVO on imaging. The NIHSS was 12 with right sided hemiplegia and slurred speech. A 5F pigtail catheter was placed in the aortic arch in the hybrid interventional (Alphenix $^{\mathrm{TM}}$ ) and CT (Genesis ${ }^{\mathrm{TM}}$ Aquilion One) suite (Canon Medical Systems, Tustin, CA). A 2-second, intra-aortic injection was performed with $20 \mathrm{ml}$ of $30 \%$ contrast-saline mixture at $10 \mathrm{ml} / \mathrm{s}$. Simultaneous triggering of the continuous CT volumetric acquisition was performed with a peak of $80 \mathrm{KVP}$ at a constant $320 \mathrm{mAmp}$. Volumetric angiography reconstruction of the data set was performed at $0.3 \mathrm{~s}$ interval $(320$ images, $0.5 \mathrm{~mm}$ thickness with $16 \mathrm{~cm}$ of coverage). The 12-

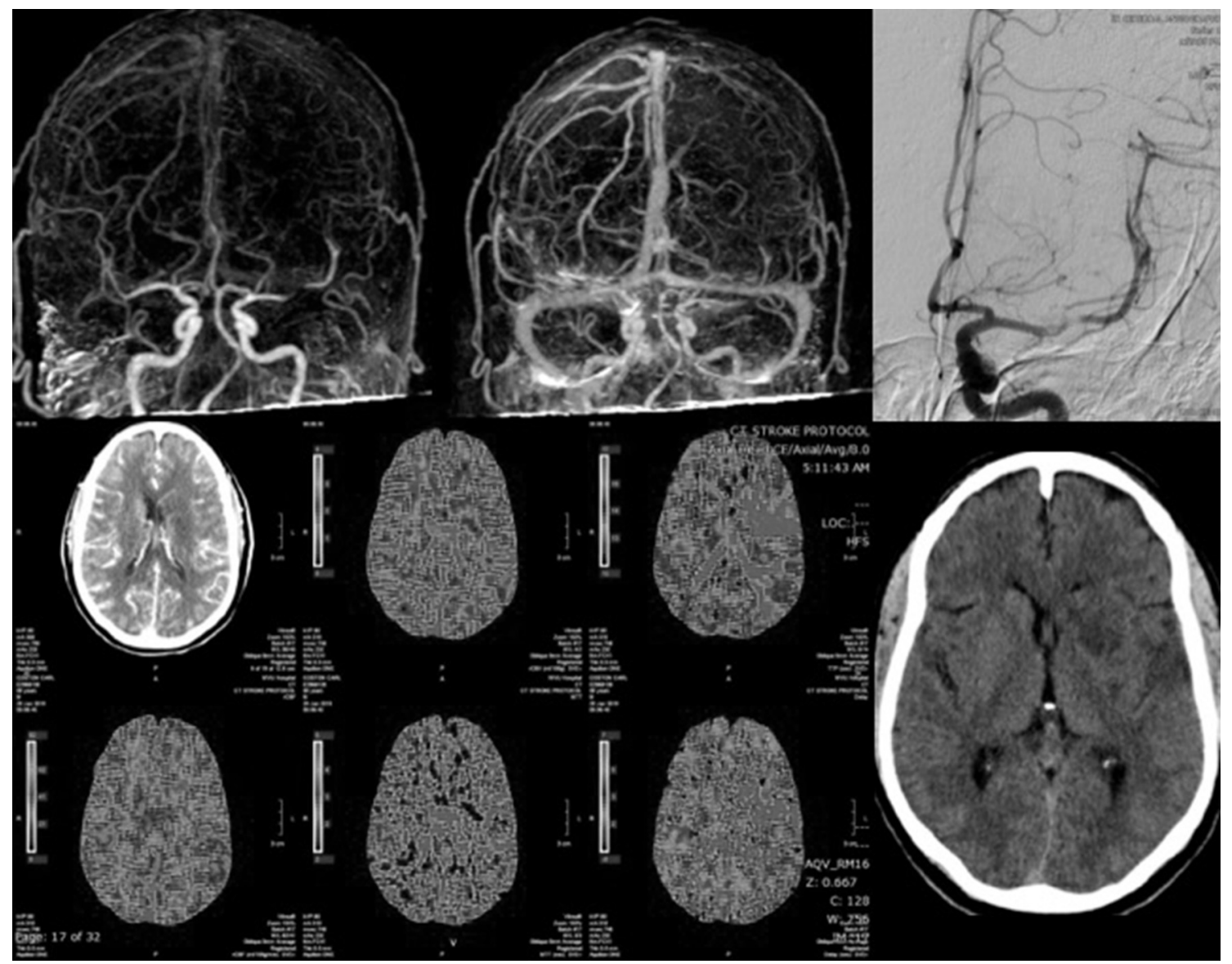

Abstract P-002 Figure 1 
second single acquisition generated a non-contrast CT of the brain, multiphase angiography at 3 frames/second and wholebrain perfusion imaging (figure 1). The non-contrast CT showed patchy infarcts. The angiography showed a sub-occlusive left MCA thrombus with good collaterals. The perfusion imaging showed matched defects corresponding to the infarcts but no significant penumbral tissue. A standard selective left internal carotid artery catheter angiography confirmed the subocclusive thrombus and good collaterals. Repeat on table clinical exam had improved from admission. Given the sub-occlusive thrombus, good collaterals and no at-risk ischemic tissue on perfusion - thrombectomy was not performed.

Conclusion Rapid assessment of suspected LVO with simultaneous treatment is feasible in select stroke patients, e.g. transfers, using a hybrid CT-Angiography suite and direct intraaortic diluted contrast injection. An intra-arterial injection gives superior signal-to-noise ratio and temporal resolution than intravenous injection. Immediate thrombectomy following the acquisition in the same room can shorten door to recanalization times.

Disclosures A. Rai: 2; C; Stryker, Microvention, Cerenovus. A. Tarabishy: None. S. Boo: 2; C; STRYKER.

\section{P-003 A NOVEL TOOL FOR THE PREDICTION OF CLINICAL OUTCOMES FOLLOWING MECHANICAL THROMBECTOMY}

S Nayak*, H Wright, A Bazarova, M Raseta. Royal Stoke University Hospitals, Stoke-onTrent, UK

\subsection{6/neurintsurg-2019-SNIS.39}

Background and purpose The purpose of this work was to develop a robust and user-friendly tool-kit to aid decisionmaking regarding Mechanical Thrombectomy (MT), based on easily-available patient variables that could accurately predict long-term functional outcome following MT. The aim of this study was to test the accuracy of four models developed to predict four types of post-treatment outcome.

Methods Data from patients with anterior circulation stroke who underwent MT between October 2009 and January 2018 $(n=240)$ were identified from our Mechanical Thrombectomy Database. Four models were developed from the data to predict the four outcomes of interest. The patient explanatory variables were age, sex, initial NIHSS, ASPECTS, Collateral Score and Glasgow Coma Scale score.

Model 1: Prediction of survival: mRS score of 0-5 (alive) or 6 (dead).

Model 2: Prediction of good/poor outcome: mRS score of 0-3 (good), or 4-6 (poor).

Model 3: Prediction of good/poor outcome: mRS score of 0-2 (good), or 3-6 (poor).

Model 4: Prediction of mRS category: mRS score of 0-2 (no disability), 3 (minor disability), 4-5 (severe disability) or 6 (dead).

The accuracy and discriminative power of each predictive model were tested.

Results Prediction of survival was $87 \%$ accurate (area under the curve 0.87). Prediction of good/poor outcome was $91 \%$ accurate (area under the curve 0.94) for Model 2 and 95\% accurate (area under the curve 0.98) for Model 3. Prediction of mRS category was $74 \%$ accurate, and increased to $95 \%$ using the 'one-score-out rule'.

Conclusion This novel tool-kit is easy to use and provided accurate estimations of outcome. It can be used to aid decision making and consent for mechanical thrombectomy.

Disclosures S. Nayak: None. H. Wright: None. A. Bazarova: None. M. Raseta: None.

\section{P-004 THROMBUS CLASSIFICATION WITH TARGETED MAGNETIC NANOPARTICLES}

${ }^{1} \mathrm{C}$ Eskey*, ${ }^{2}$ W Wells, ${ }^{1} \mathrm{D}$ Schartz, ${ }^{2} \mathrm{C}$ Jackson, ${ }^{1} \mathrm{~J}$ Weaver. ${ }^{1}$ Radiology, Dartmouth Hitchcock Medical Center, Lebanon, NH; ${ }^{2}$ Pathology, Dartmouth Hitchcock Medical Center, Lebanon, NH

\subsection{6/neurintsurg-2019-SNIS.40}

Purpose and introduction Thromboembolic material producing large vessel occlusion of the cerebral arteries varies substantially in composition and these differences affect the ability of both intravenous thrombolysis and mechanical thrombectomy to achieve rapid arterial recanalization. The binding of targeted magnetic nanoparticles may offer a means of noninvasively determining clot composition. We hypothesize that binding of targeted magnetic nanoparticles to thrombus is measurable and varies with clot composition.

Materials and methods Thromboembolic material was extracted from 10 patients during emergent mechanical thrombectomy for large vessel occlusion in ICA, MCA, or basilar arteries. The nominal source of the thromboembolic material and time from onset to clot retrieval were recorded. Each sample was divided and sent both for standard histopathologic evaluation and for in vitro assessment by magnetic spectroscopy of nanoparticle Brownian rotation (MSB). The thromboembolic material was incubated in a solution of magnetic nanoparticles that had been coated with antibodies directed toward thrombin. Nanoparticle binding was measured using magnetic relaxometry.

Results All clots bound measurable quantities of the thrombindirected magnetic nanoparticles. $2 / 10$ of the samples were composed of $>90 \%$ fibrin material. These two samples demonstrated much higher than expected nanoparticle binding. The other samples had proportions of thrombus that ranged from 50 to $60 \%$. Among these samples there was a significant correlation between nanoparticle weight and $\mathrm{RBC}$ content $\left(\mathrm{R}^{2}\right.$ $=0.70, \mathrm{p}<0.04)$ and a lesser association between nanoparticle relaxation and $\mathrm{RBC}$ content $\left(\mathrm{R}^{2}=0.49, \mathrm{p}<0.12\right)$.

Discussion Thrombin-directed nanoparticles bound thrombinrich thromboembolic material roughly in proportion to the RBC content, as expected, confirming an affinity of these particles for this component of the clot. However, samples consisting almost exclusively of fibrin bound even higher levels of the nanoparticles suggesting the presence of an additional binding mechanism.

Disclosures C. Eskey: None. W. Wells: None. D. Schartz: None. C. Jackson: None. J. Weaver: None. 